\title{
Força muscular respiratória de jogadores de basquetebol em cadeira de rodas: comparação entre os valores obtidos e preditos
}

\author{
Respiratory muscle strength of wheelchair basketball players: comparison between the \\ obtained and predicted values
}

Fuerza muscular respiratoria de jugadores de baloncesto en silla de ruedas: comparación entre los valores obtenidos y predecidos

Núbia Cristina Braga da Silva ${ }^{1}$, Allan Oliveira de Lira ${ }^{1}$, Antônio Gabriel Pantoja Silva Santos ${ }^{1}$, Júlio César Veiga Pena ${ }^{1}$, Raphael do Nascimento Pereira ${ }^{1}$, Valéria Marques Ferreira Normando ${ }^{1 \star}$.

\section{RESUMO}

Objetivo: Avaliar a força muscular respiratória (FMR) de jogadores de basquetebol em cadeira de rodas (BCR) com lesão medular traumática (LMT) e comparar os valores obtidos aos preditos. Métodos: Trata-se de estudo de inquéritos, quantitativo, unicêntrico. Dezesseis atletas do gênero masculino foram divididos em dois grupos: $L M T, n=8$, e; sem $L M T, n=8$. Os dois grupos foram avaliados quanto as variáveis antropométricas e a FMR, esta por meio da manovacuômetria para mensuração das pressões inspiratória (PImáx) e expiratória máxima (PEmáx). Resultados: A amostra foi constituída por dezesseis atletas do gênero masculino, com idade entre 34,75 $\pm 8,55$ anos para o grupo LMT e 24,50 $\pm 5,37$ anos para o grupo sem LMT. Ambos os grupos apresentaram valores de PImáx obtido superior ao predito. Os valores de PEmáx obtido pelo grupo sem LMT foi superior ao predito e ao valor obtido pelo grupo com LMT. O percentual alcançado na PEmáx predita do grupo sem LMT foi superior ao do grupo com LMT. Conclusão: Apesar dos benefícios da prática regular do BCR observados sobre a força muscular inspiratória (FMI) dos atletas, estes efeitos parecem não se aplicar à força muscular expiratória (FME) dos sujeitos com LMT.

Palavras-chave: Pressões respiratórias máximas, Traumatismos da medula espinal, Paratletas.

\section{ABSTRACT}

Objective: To evaluate the respiratory muscle strength (RMS) of basketball players in wheelchairs (BPW) with traumatic spinal cord injury ( $\mathrm{SCl}$ ) and to compare the values obtained with the predicted ones. Methods: This is a single-center, quantitative, survey study. Sixteen male athletes were divided into two groups: $\mathrm{SCl}, \mathrm{n}=8$, and; without $\mathrm{SCl}, \mathrm{n}=8$. Both groups were evaluated for anthropometric variables and the RMS, using the manovacuometer to measure the maximum inspiratory (MIP) and maximum expiratory (MEP) pressures. Results: The sample consisted of sixteen male athletes, aged between $34.75 \pm 8.55$ years for the SCl group and $24.50 \pm 5.37$ years for the group without SCl. Both groups had MIP values obtained higher than predicted. The MEP values obtained by the group without $\mathrm{SCl}$ were higher than predicted and the value obtained by the group with $\mathrm{SCl}$. The percentage reached in the predicted MEP of the group without $\mathrm{SCl}$ was higher than that of the group with SCl. Conclusion: Despite the benefits of regular BPW practice observed on athletes' inspiratory muscle strength (IMS), these effects do not seem to apply to the expiratory muscle strength (EMS) of subjects with $\mathrm{SCl}$.

Keywords: Maximum respiratory pressures, Spinal cord injuries, Parathletes.

1 Universidade do Estado do Pará (UEPA), Belém - PA.

*E-mail: valeriafisio@gmail.com 


\section{RESUMEN}

Objetivo: Evaluar la fuerza de la musculatura respiratoria (FMR) de jugadores de baloncesto en silla de ruedas (BSR) con lesión medular traumática (LMT) y comparar los valores obtenidos con los predichos. Métodos: Este es un estudio de encuesta cuantitativo, unicentrico. Dieciséis atletas masculinos se dividieron en dos grupos: LMT, $n=8 \mathrm{y} ; \sin L M T, n=8$. Ambos grupos fueron evaluados para las variables antropométricas y la FMR, utilizando el manovacuómetro para medir las presiones inspiratoria (PImáx) y espiratoria máxima (PEmáx). Resultados: La muestra estuvo conformada por dieciséis atletas masculinos, con edades entre 34,75 $\pm 8,55$ años para el grupo LMT y 24,50 $\pm 5,37$ años para el grupo sin LMT. Ambos grupos obtuvieron valores de PImáx superiores a los previstos. Los valores de PEmáx obtenidos por el grupo sin LMT fueron superiores a los previstos y al valor obtenido por el grupo con LMT. El porcentaje alcanzado en el PEmáx previsto del grupo sin LMT fue superior al del grupo con LMT. Conclusión: A pesar de los beneficios de la práctica regular de BSR observados en la fuerza de los músculos inspiratorios (FMI) de los atletas, estos efectos no parecen aplicarse a la fuerza de los músculos espiratorios (FME) de los sujetos con LMT.

Palabras clave: Presiones respiratorias máximas, Traumatismo espinal, Paratletas.

\section{INTRODUÇÃO}

Hodiernamente, é insofismável que a lesão da medula espinhal continua sendo algo assíduo, tendo uma incidência, por exemplo, de aproximadamente 16 a 26 milhões de brasileiros por ano. Na maioria dos casos a lesão medular é oriunda de episódios traumáticos, como em casos de compressão, laceração, distração ou cisalhamento da medula espinal. Assim, as consequências do evento supracitado são abrangentes, envolvendo o sistema cardiovascular, respiratório, gastrointestinal, hematológico, neurológico, geniturinário e tegumentar. Logo, todo o processo de tratamento e reabilitação destes pacientes inclui vários profissionais e modalidades, como a prática esportiva, a qual já se mostrou eficaz tanto na melhora de aspectos físicos quanto mentais (ECKERT MJ e MARTIN MJ, 2017; ROUANET C, et al., 2017; SKUCAS K, et al., 2014).

Atualmente, o número de pessoas com deficiência que procuram praticar algum esporte vem crescendo gradativamente a cada dia. Além de reduzir o risco de comorbidades, o esporte possibilita a otimização da força, resistência e flexibilidade, bem como auxilia no processo de reintegração na sociedade. Desta forma, o basquetebol em cadeiras de rodas (BCR) tem grande destaque, o qual tem períodos de baixa e alta intensidade, sendo praticado por pessoas com amputações, poliomielite, lesões medulares entre outras disfunções (SANTOS MVL, et al., 2016; CORRÊA BDC, et al., 2018).

Por meio de uma análise histórica lacônica, percebe-se que a inclusão das pessoas com deficiências no âmbito esportivo só ocorreu e se disseminou a partir da Segunda Guerra Mundial, quando os veteranos de guerra, os quais muitos apresentavam lesão medular, foram recebidos como heróis e, gradativamente, a prática esportiva foi sendo introduzida para as pessoas com deficiência como medida terapêutica. Assim, o BCR foi uma das modalidades esportivas de destaque, estando presente em 1960 na primeira Paraolimpíada, na cidade de Roma (COSTA GR, 2017).

No contexto brasileiro, o BCR é o esporte pioneiro desenvolvido por pessoas com deficiência, sendo praticado desde o final da década de 1950. O BCR foi introduzido no Brasil, primeiramente, no Rio de Janeiro e São Paulo por Sergio Seraphim Del Grande e Robson Sampaio de Almeida, os quais realizaram um programa de reabilitação nos Estados Unidos (BEGOSSI TD, et al., 2019).

Desta forma, nos dias atuais, o BCR é um dos esportes paraolímpicos mais praticados e, visando a igualdade entre as equipes, o BCR apresenta as suas próprias regras acerca da formação das equipes, considerando, sobretudo, a funcionalidade de cada atleta (MARSZAŁEK J, et al., 2019).

Assim, a classificação funcional dos atletas segue critérios que avaliam amplitude, força e coordenação das funções de tronco, membros inferiores (MMII), membros superiores (MMSS) e das mãos. Portanto, a classificação funcional decorre do grau de comprometimento do atleta, podendo variar entre 1.0, que representaria uma lesão alta e, por conseguinte, um maior comprometimento, até 4.5 (CORRÊA W, 2016). 
Logo, quanto menor esta pontuação, maior e mais alto será o comprometimento motor, além disso, em quadra a somatória dos pontos dos cinco jogadores não pode exceder 14 pontos, pois representaria uma vantagem sobre a equipe adversária, haja vista que uma das equipes estaria com um maior número de jogadores com menos comprometimento funcional (GIL SM, et al., 2015).

Dentre as pessoas com deficiências físicas, os indivíduos com lesão medular apresentam importantes alterações da função respiratória relacionadas com o nível e com o tipo (incompleta ou completa) da lesão (BERLOWITZ DJ, et al., 2016; MACEDO FS, et al., 2017). Entres as alterações respiratórias presente na lesão medular destacam-se a atelectasia, pneumonia e insuficiência respiratória, além disso, lesões acima de C4 representam consequências dantescas para os músculos respiratórios, situação na qual o paciente necessitará de ventilação mecânica. Ademais, $65 \%$ dos pacientes com lesão medular em níveis mais baixos, como entre T1 e T12, também podem ter complicações respiratórias graves, uma vez que tal seguimento é responsável pela inervação de músculos importantes respiratório, como os intercostais (HAGEN EM, 2015; NETTER FH, 2015).

Para tanto, a avaliação da força muscular respiratória (FMR) é importante para melhor julgar o possível grau de disfunção dos músculos respiratórios destes indivíduos, uma vez que estes músculos representam o fator fisiomecânico mais importante para uma ventilação pulmonar adequada (MORENO MA, et al., 2012; OLIVEIRA AG, et al., 2017). Assim, manovacuometria, indicada como um meio simples, útil e não invasivo de avaliar as pressões inspiratória máxima (PImáx) e expiratória máxima (PEmáx), ela é capaz de predizer o índice da força dos músculos inspiratórios (FMI) e expiratórios (FME) (SANTOS RMGD, et al., 2017).

Desta forma, tanto a PImáx quanto a PEmáx podem ser medidas na boca por meio de um manovacuômetro. Os resultados destas pressões e a interpretação correta destas auxiliam na prática clínica de maneira significativa, pois uma possível fraqueza da musculatura respiratória pode ser excluída se a PImáx e PEmáx estarem adequada para o sexo e idade do paciente. Destarte, essas medidas são úteis para distinguir entre uma real fraqueza do diafragma ou outros músculos inspiratórios e uma fraqueza dos músculos abdominais (ANDRADE CR, et al., 2014). Igualmente, visando uma maior precisão nos dados da manovacuometria, há disponível na literatura equações para o cálculo de PImáx e PEmáx em função da idade e de acordo com o gênero (OLIVEIRA JJJD, et al., 2016).

Apesar da importância que o BCR tem como alternativa para a prática esportiva de pessoas com deficiências físicas, ainda se encontra lacunas com relação a estudos que descrevem acerca dos valores das pressões respiratórias máximas (PRM) para esta população (MORENO MA, et al., 2012).

Destarte, partindo do pressuposto que o BCR é um esporte competitivo e que exige altas demandas fisiológicas, sejam elas relacionadas ao desempenho aeróbico ou anaeróbico, a avaliação da FMR destes atletas é de suma importância, uma vez que a fraqueza dos músculos respiratórios está intrinsicamente ligada à redução da Capacidade Vital Forçada (CVF), da Ventilação Voluntária Máxima (VVM) e do Volume Expiratório Forçado no 1․ Segundo $\left(\mathrm{FEV}_{1}\right)$. À vista disso, a fraqueza muscular respiratória pode afetar a tolerância aos exercícios, afetando diretamente a performance durante os jogos (ITURRICASTILLO A, et al., 2016; WANG X, et al., 2020).

Logo, considerou-se favorável pesquisar a FMR em atletas jogadores de BCR, a fim de comparar o valor obtido ao predito, por meio das equações propostas na literatura para a população brasileira (OLIVEIRA JJJD, et al., 2016). No intuito de verificar se são correspondentes, subestimados ou superestimados, devido à prática esportiva. Dessa forma, objetivou-se comparar os valores obtidos e preditos, a partir da avaliação da força muscular respiratória de jogadores de basquetebol em cadeira de rodas.

\section{MÉTODOS}

Trata-se de um estudo de inquéritos, quantitativo, unicêntrico realizado de acordo com as normas de conduta em pesquisa experimental com seres humanos descritas na resolução 466/12 do Conselho Nacional de Saúde. Foi aprovado pelo Comitê de Ética em Pesquisa da Universidade do Estado do Pará, sob o parecer número 1.111.508/15. Neste, todos assinaram previamente um termo de consentimento livre e esclarecido (TCLE). 
Foram convidados quarenta atletas de BCR, de ambos os gêneros, procedentes do Clube dos Deficientes Físicos do Pará - "All Star Rodas", praticantes, de forma regular, de BCR há mais de três meses, com idade entre 19 e 50 anos, vínculo efetivo com o Clube "All Star Rodas", não tabagistas, sem presença de alterações cardiovasculares, hormonais, respiratórias, ortopédicas e/ou neuromusculares diagnosticadas que impedissem a realização dos testes.

Após esta seleção, dezesseis atletas do gênero masculino, aceitaram participar do estudo, os quais foram divididos em dois grupos: lesão medular traumática (LMT), $n=8$, sendo 5 lesões completas e 3 incompletas entre os níveis T6 e L5 e; sem lesão medular traumática (sem LMT), $n=8$, incluindo malformação congênita, amputação em membros inferiores (MMII) e alterações ortopédicas de MMII. Outrossim, todos os procedimentos foram realizados no ginásio do Comando da Guarda Municipal de Belém do Pará, localizado na Av. Pedro Álvares Cabral, 1400, Belém, Pará.

Além disso, em relação aos procedimentos experimentais, primeiramente realizou-se uma triagem por meio de entrevista, com o preenchimento de um questionário com perguntas referentes à aspectos pessoais, da deficiência física e condições de treino dos mesmos, seguindo os critérios de inclusão e exclusão. Posteriormente, foram verificadas as variáveis antropométricas e respiratórias. Os procedimentos avaliativos obedeceram ao protocolo seguido por Pereira RN (2013) e foram esclarecidos aos voluntários do estudo.

Assim, para a avaliação da FMR foram medidas as PImáx e PEmáx, utilizando um manovacuômetro digital, composto por um transdutor de pressão MVD-300® (Microhard System, Globalmed, São Paulo, Brasil), com capacidade de $\pm 300 \mathrm{cmH}_{2} \mathrm{O}$, conectado a um sistema com duas válvulas unidirecionais, uma inspiratória e outra expiratória.

As PImáx e PEmáx foram obtidas a partir do volume residual (VR) e da capacidade pulmonar total (CPT), respectivamente, com os voluntários sentados de forma ereta (com $90^{\circ}$ de flexão do quadril), usando um clipe nasal para evitar escape de ar pelas narinas, e um bocal plástico e rígido com um pequeno orifício para prevenir os efeitos do fechamento da glote durante as manobras conectado ao manovacuômetro.

Realizou-se no mínimo cinco manobras máximas, sendo três aceitáveis e duas reprodutíveis (com diferença inferior a $10 \%$ entre elas), com intervalo de um minuto entre cada, sustentando o esforço inspiratório e expiratório por ao menos um segundo, com registro dos maiores valores obtidos para as análises (MORENO MA, et al., 2012; SANTOS RMGD, et al., 2017).

Neste estudo, os valores da PImáx e PEmáx obtidos em cada voluntário foram comparados com os valores preditos pelas seguintes equações (Para Homens: PImáx: $y=-0,80 \times$ idade $+155,3$; erro-padrão da estimativa $=17,3$; PEmáx: $y=-0,81 \times$ idade $+165,3$; erro-padrão da estimativa $=15,6)($ OLIVEIRA JJJD, et al., 2016).

Todos os dados desta pesquisa foram apresentados em médias e desvios-padrões, sendo adotada a significância de 5\%. A análise estatística foi realizada com o auxílio do programa BioEstat, versão 5.0, no qual, inicialmente fez-se uma análise descritiva dos dados. Em seguida, foi utilizado o teste de Shapiro-Wilk para análise da normalidade na distribuição dos elementos estudados, e, a partir da constatação da normalidade, optou-se pelo teste T de Student para as análises comparativas.

\section{RESULTADOS}

A Tabela 1 descreve as características dos grupos, por meio da média \pm desvio padrão para as variáveis investigadas. A amostra foi constituída por dezesseis atletas do gênero masculino, com idade entre $34,75 \pm$ 8,55 anos para o grupo LMT e 24,50 $\pm 5,37$ anos para o grupo sem LMT $(p<0.05)$.

Para a classificação funcional pela IWBF (International Wheelchair Basketball Federation), $50 \%$ da amostra $(n=8)$ foram pontuados entre 1 e $1.5 ; 6,25 \%(n=1)$ entre 2 e $2.5 ; 25 \%(n=4)$ entre 3 e 3.5 e; $18,75 \%$ $(n=3)$ entre 4 e 4.5. De maneira geral, $56,25 \%(n=9)$ da amostra pertenciam a Categoria A, que representa os atletas que obtiveram a pontuação entre 1,0 a 2,5, os quais não apresentam o controle do quadril; enquanto $43,75 \%(n=7)$ pertenciam a categoria $B$, que inclui os atletas que obtiveram a pontuação entre 3,0 a 4,5, e que apresentam o controle do quadril. 
Em relação à etiologia da deficiência motora encontrada, 50\% da amostra $(n=8)$ são portadores de LMT entre os níveis de T6 - L5; 31,25\% ( $n=5)$ apresentam malformação congênita; 12,5\% ( $n=2)$ são de amputação em MMII e; 6,25\% (n=1) são de alterações ortopédicas de MMII.

Tabela 1 - Características dos grupos, apresentadas em média (desvio padrão).

\begin{tabular}{lccc}
\hline Variáveis & Grupo LMT & Grupo sem LMT & P valor \\
\hline $\mathbf{N}$ & 8 & 8 & - \\
\hline Idade (anos) & $34.75(8.55)$ & $24.50(5.37)$ & $<0.05$ \\
\hline Massa corporal (kg) & $70.83(8.38)$ & $54.43(7.06)$ & $<0.05$ \\
\hline Estatura (cm) & $166.25(8.73)$ & $147.13(21.05)$ & $<0.05$ \\
\hline Tempo de prática esportiva (anos) & $5.13(3.14)$ & $5.75(3.62)$ & 0.72 \\
\hline Volume de treino (horas/semana) & $15.88(5.69)$ & $17.25(5.09)$ & 0.67 \\
\hline Frequência semanal de treino (N) & $4.25(1.04)$ & $4.50(0.93)$ & 0.67 \\
\hline Classificação funcional pela IWBF (N) & & & \\
\hline 1 - 1.5 & 7 & 1 & - \\
2 - 2.5 & 0 & 1 & - \\
3 - 3.5 & 1 & 3 & - \\
4 - 4.5 & 0 & 3 & - \\
\hline Etiologia da deficiência motora (N) & & & - \\
\hline LMT (T6 - L5) & 8 & 0 & - \\
Malformação congênita & 0 & 5 & - \\
Amputação em MMII & 0 & 2 & \\
Alterações ortopédicas de MMII & 0 & 1 & \\
\hline
\end{tabular}

Legenda: $\mathrm{N}$ = tamanho da amostra. IWBF = "International Wheelchair Basketball Federation". LMT = lesão medular traumática. (T6 - L5) = intervalo dos níveis de lesões medulares encontradas. MMII = membros inferiores. Fonte: Silva NCB, et al., 2021.

Com a descrição dos valores referentes à FMI e FME, observou-se que a média da PImáx obtida e predita, respectivamente, em $\mathrm{cmH} 2 \mathrm{O}$, no grupo LMT foi 170,75 $\pm 33,98$ e 127,50 \pm 6,84 e; no grupo sem LMT foi 183,63 $\pm 32,92$ e 135,70 $\pm 4,30$. A média da PEmáx obtida e predita, respectivamente, em $\mathrm{cmH} 2 \mathrm{O}$, no grupo LMT foi $162,75 \pm 76,73$ e 193,45 \pm 6,92; no grupo sem LMT foi $256 \pm 30,53$ e 185,15 $\pm 4,35$ (Tabela 2).

Tabela 2 - Valores obtidos e preditos referentes à força muscular inspiratória e expiratória. Dados apresentados em média (desvio padrão).

\begin{tabular}{lcc}
\hline Variáveis & Grupo LMT & Grupo sem LMT \\
\hline Força muscular inspiratória & & \\
\hline PImáx predita $\left(\mathrm{cmH}_{2} \mathrm{O}\right)$ & $127.50(6.84)$ & $135.70(4.30)$ \\
PImáx obtida $\left(\mathrm{cmH}_{2} \mathrm{O}\right)$ & $170.75^{*}(33.98)$ & $183.63^{*}(32.92)$ \\
Percentual alcançado $(\%)$ & $133.96(25.64)$ & $135.33(23.52)$ \\
\hline Força muscular expiratória & & \\
\hline PEmáx predita $\left(\mathrm{cmH}_{2} \mathrm{O}\right)$ & $193.45(6.92)$ & $185.15(4.35)$ \\
PEmáx obtida $\left(\mathrm{cmH}_{2} \mathrm{O}\right)$ & $162.75(76.73)$ & $256.00 * \dagger(52.83)$ \\
Percentual alcançado $(\%)$ & $84.11(39.24)$ & $138.65 \dagger(30.53)$ \\
\hline
\end{tabular}

Legenda: $L M T=$ lesão medular traumática. PImáx $=$ pressão inspiratória máxima. PEmáx $=$ pressão expiratória máxima. ${ }^{*}=\mathrm{P}<0.05$ (diferença em relação ao valor predito). $\dagger=\mathrm{P}<0.05$ (diferença em relação ao Grupo LMT). Fonte: Silva NCB, et al., 2021. 


\section{DISCUSSÃO}

Neste estudo, pode-se observar que os valores da PImáx obtida em ambos os grupos se apresentaram semelhantes, e estatisticamente superiores aos valores preditos. Assim, a literatura apresenta algumas explicações para justificar tal fato, entre elas a prática regular do BCR e o nível da lesão medular, como destacado abaixo.

Sugere-se que este achado seja efeito da prática regular do BCR, a qual de acordo com os dados apresentados na caracterização da amostra equivale há cerca de cinco anos. O BCR utiliza amplamente os membros superiores (MMSS) e a movimentação do tronco durante a propulsão da cadeira de rodas, bem como nos arremessos realizados durante o jogo (PEREIRA RN, 2013). Além disso, durante um jogo de BCR há uma predominância de movimentos intensos e vigorosos, o que por sua vez expõem uma grande demanda sobre o sistema cardiorrespiratório (SERON BB, et al., 2019).

Ademais, outro fator que justifica a igualdade da PImáx obtida em ambos os grupos se deve pelo fato de que o nível da lesão medular permite a preservação do diafragma, inervado pelas raízes nervosas de C3 e $\mathrm{C} 5$, bem como o funcionamento da musculatura respiratória acessória, a qual envolve alguns grupos musculares presentes nos MMSS e cintura escapular. Assim, os músculos supracitados participam da estabilização, posicionamento e execução de movimentos dos MMSS e do tronco, além de estarem envolvidos no processo de inspiração forçada durante a prática do BCR (ANTONELLI CB, et al., 2020; PEREIRA RN, et al., 2016; MORENO MA, et al., 2012; PEREIRA RN, 2013; HAUBERT LL, et al., 2020).

Moreno MA, et al. (2012) avaliaram a influência da prática regular de Rugby e BCR em sujeitos tetraplégicos e paraplégicos, respectivamente, mensurando as PRM, por meio de um manovacuômetro tipo anaeróide sugerindo que, a prática destes esportes exerceu adaptações benéficas sobre a FMR dos atletas com LMT, apesar de outros autores, como a Manchur VF e Volski V (2017) destacarem que jogadores de BCR com lesão medular apresentam uma menor força, agilidade e potência aeróbica.

Neste estudo, os voluntários apresentaram nível da LMT entre T6 e L5, tendo, provavelmente, disfunção inspiratória em virtude da diminuição da funcionalidade dos músculos intercostais, os quais possuem inervação oriunda de raízes medulares em níveis de T1 a T11. Logo, uma atenuação da funcionalidade dos intercostais ocasiona uma diminuição do diâmetro anteroposterior lateral do tórax e, por conseguinte, um volume inspiratório menor (GALEIRAS VR, et al., 2013; NETTER FH, 2015).

Pereira RN (2013) avaliou a relação entre FMR e desempenho físico de jogadores de BCR utilizando um manovacuômetro analógico para obtenção das medidas das PRM. Neste estudo, o autor descreveu que os sujeitos com LMT no nível entre T4 e L5, apresentaram modificação na função inspiratória. Logo, as alterações na funcionalidade dos músculos intercostais são capazes de gerar redução da FMI e consequente diminuição da expansibilidade torácica (FREZ AR, et al., 2014).

Neste contexto, considera-se que os principais músculos inspiratórios têm uma localização superior no tronco e são inervados pelos segmentos superiores da coluna, portanto, quanto mais alto for o nível da lesão da medula espinhal, maior o comprometimento para a musculatura inspiratória (MORENO MA, et al., 2014). Entretanto, é valido ressaltar que este estudo apontou que ambos os grupos apresentaram aumentos significativos de PImáx. Segundo Moreno MA, et al. (2012), o fato de um cadeirante com LMT treinar regularmente uma modalidade esportiva já é capaz de promover adaptações benéficas sobre a função respiratória, devido à maior exigência desta musculatura durante os treinamentos.

Em relação à PEmáx observou-se que, os valores obtidos apresentaram-se inferiores aos preditos apenas no grupo LMT, o qual pode ser atribuído ao possível acometimento funcional dos músculos abdominais, bem como ao nível da lesão.

No estudo realizado por Moreno MA, et al. (2012), quando analisados os grupos paraplégicos, os resultados apontam que, independentemente dos níveis de aptidão física, a FME foi comprometida, confirmando que o nível da lesão tem um impacto negativo sobre a mesma, o que pode ser explicado pela insuficiência dos segmentos da medula espinhal responsáveis pelos principais músculos expiratórios ativados na expiração forçada máxima. 
Pereira RN, et al. (2016) realizaram um estudo acerca da FMR com atletas de BCR, os quais foram divididos em dois grupos: com controle de tronco e sem controle de tronco. Dessa forma, o estudo evidenciou que a principal diferença entre os dois grupos estava na redução PEmáx no grupo sem controle de tronco, o qual também apresentava menor capacidade aeróbica.

A diferença supracitada encontrada talvez possa estar relacionada ao comprometimento das raízes que originam a inervação dos músculos intercostais (T1-T11), reto do abdome (T6-T12), transverso do abdome (T2-L1) e oblíquos internos e externos (T6-L1), podendo culminar em alteração da dinâmica ventilatória e modificação da biomecânica do diafragma, uma vez que os músculos abdominais possuem importante função na estabilização das vísceras abdominais, na manutenção da posição do diafragma para cima durante a expiração forçada e, principalmente na manutenção da pressão intratorácica e abdominal (MCKENZIE DC, 2012; ISHIDA H, et al., 2015).

Outrossim, os músculos abdominais como músculos respiratórios têm ação predominantemente expiratória e são inervados pelos nervos torácicos de T7 a T12 e primeiro nervo lombar, sendo, invariavelmente mais afetados que os músculos inspiratórios, independentemente do nível da LMT (MCKENZIE DC, 2012; MACHADO MGR, 2018).

De acordo com Pereira RN (2013), a manutenção da funcionalidade dos músculos abdominais está relacionada com a preservação do controle de tronco, o qual é nivelado por meio da avaliação da classificação funcional pela IWBF. Logo, quanto maior a estabilização do tronco e movimentação angular realizada com a flexão e extensão do tronco, maior é a pontuação na classificação funcional da IWBF (VANCINI RL, et al., 2018). Assim sendo sugere-se que os sujeitos do grupo sem LMT possuem melhor controle e estabilização do tronco, tendo em vista que estes têm maior pontuação na classificação funcional da IWBF. Possivelmente, também podem apresentar preservação da funcionalidade dos músculos abdominais e do tronco, fato que provavelmente influenciou para que os sujeitos deste grupo apresentassem PEmáx superior a predita, bem como significativamente superior aos valores observados no grupo LMT.

Vale salientar que os sujeitos do grupo sem LMT não apresentaram valores de PEmáx obtidos inferiores ao predito, de acordo com as equações propostas na literatura para indivíduos sem deficiências físicas (OLIVEIRA JJJD, et al., 2016).

Em relação às limitações do estudo, deve-se levar em consideração a interpretação dos resultados apresentados, visto que as equações de predição utilizadas foram desenvolvidas para indivíduos sem deficiências físicas, o que pode superestimar a diferença entre os valores preditos e obtidos em ambos os grupos.

Entretanto, os resultados apresentados devem ser um ponto de partida para futuras investigações acerca da FMR de jogadores de BCR com LMT. Sugere-se portanto, o prosseguimento do presente estudo com uma amostra maior, bem como o desenvolvimento de equações preditas para pessoas com deficiências físicas contribuindo assim, à comunidade científica.

\section{CONCLUSÃO}

O impacto da prática de basquetebol em cadeira de rodas, revelou efetividade na manutenção da PImáx acima dos valores preditos, em ambos os grupos, atribuindo-se principalmente à maior exigência das referidas musculaturas durante os treinamentos. Porém, diante das adaptações limitadas pelo nível da lesão, o que impacta com maior facilidade à função dos músculos expiratórios, não observou-se significânica nos dados da PEmáx, podendo associar-se ao déficit do controle de tronco nos atletas com LMT. Portanto, os resultados do presente estudo evidenciam a necessidade de uma maior atenção sobre 0 treino de equilíbrio de tronco dos atletas de BCR com LMT, visando, assim, otimizar possíveis benefícios deste esporte sobre a função respiratória.

\section{AGRADECIMENTOS}

Aos atletas do "All Star Rodas do Pará" por participarem deste estudo. 


\section{REFERÊNCIAS}

1. ANDRADE CR, et al. Evaluation of respiratory muscle strength in mouth breathers: clinical evidences. International archives of otorhinolaryngology, 2014; 18(3): 289-293.

2. ANTONELLI CB, et al. Effects of Inspiratory Muscle Training with Progressive Loading on Respiratory Muscle Function and Sports Performance in High-Performance Wheelchair Basketball Athletes: A Randomized Clinical Trial. International journal of sports physiology and performance, 2020: 15(2): 238-242.

3. BEGOSSI TD, et al. O basquetebol em cadeira de rodas. Revista Brasileira de Educação Física e Esporte, 2019; 33(1): 29-37.

4. BERLOWITZ DJ, et al. Respiratory problems and management in people with spinal cord injury. Breathe, 2016; 12(4): 328-340.

5. CORREA BDC, et al. Avaliação e classificação da capacidade física aeróbia de atletas de basquetebol em cadeira de rodas. Revista Brasileira de Ciências do Esporte, 2018; 40 (2): 163-169.

6. CORREA W. Basquete sobre rodas: análise da ansiedade Pré-competitiva. Revista da Associação Brasileira de Atividade Motora Adaptada ,2016; 17(2): 31-36.

7. COSTA GR. Basquetebol em cadeira de rodas e a lesão medular. Episteme Transversalis, 2017; 4(1).

8. ECKERT MJ, MARTIN MJ. Trauma: lesão da medula espinhal. Surgical Clinics, 2017; 97 (5): 1031-1045.

9. FREZ AR, et al. Perfil funcional de atletas de basquetebol com traumatismo da medula espinal de acordo com a CIF. Revista Brasileira de Medicina do Esporte, 2014; 20(1): 78-81.

10. GALEIRAS VR, et al. Respiratory management in the patient with spinal cord injury. BioMed research international, 2013; 2013: 168757.

11. GIL SM, et al. A classificação funcional e o desempenho em testes de campo em jogadores de basquete em cadeira de rodas. Journal of Human Kinetics, 2015; 46 (1): 219-230.

12. HAGEN EM. Acute complications of spinal cord injuries. World journal of orthopedics, 2015; 6(1): 17.

13. HAUBERT LL, et al. Effect of reverse manual wheelchair propulsion on shoulder kinematics, kinetics and muscular activity in persons with paraplegia. The journal of spinal med medicine, 2020; 43 (5), 594-606.

14. ISHIDA H, et al. Correlation between abdominal muscle thickness and maximal expiratory pressure. J Ultrasound Med, 2015; 34(11): 2001-2005.

15. ITURRICASTILLO A, et al. Physiological responses between players with and without spinal cord injury in wheelchair basketball small-sided games. Spinal Cord, 2016; 54(12): 1152-1157.

16. MACEDO FS, et al. Novas perspectivas de fisioterapia respiratória em lesão medular - uma revisão sistemática. Acta Paulista de Enfermagem, 2017; 30(5): 554-564.

17. MACHADO MGR. Bases da fisioterapia respiratória: terapia intensiva e reabilitação. 2 ed. Rio de Janeiro: Guanabara Koogan, 2018.

18. MANCHUR VF, VOLSKI V. Avaliação de capacidades físicas de atletas de basquetebol em cadeiras de rodas. Rev Assoc Bras Ativ Mot Adapt, 2017; 18(2): 119-130 2017.

19. MARSZAŁEK J, et al. Laboratory and non-laboratory assessment of anaerobic performance of elite male wheelchair basketball athletes. Frontiers in psychology, 2019; 10: 514.

20. MCKENZIE DC. Respiratory physiology: adaptations to high-level exercise. Br J Sports Med, 2012; 46: 38-384.

21. MORENO MA, et al. Camargo TMD, Barros RMLD. Inspiratory muscle strength in subjects with tetraplegia: viability of evaluation through the measurement of maximal inspiratory pressure. Fisioterapia em Movimento, 2014; $27(3)$ : 371377.

22. MORENO MA, et al. Effects of wheelchair sports on respiratory muscle strength and thoracic mobility of individuals with spinal cord injury. American journal of physical medicine \& rehabilitation, 2012; 91(6): 470-477.

23. NETTER FH. Atlas de anatomia humana. 6 ed. Rio de Janeiro: Elsevier, 2015.

24. OLIVEIRA AG, et al. A efetividade do treinamento muscular respiratório com powerbreathe em atletas de basquete. Fisioterapia em Ação-Anais eletrônicos, 2017: 21-32.

25. OLIVEIRA JJJD, et al. Efeito da fisioterapia ambulatorial pós-operatória sobre a capacidade funcional e a força muscular respiratória em pacientes submetidos à cirurgia bariátrica. ABCD. Arquivos Brasileiros de Cirurgia Digestiva, 2016; 29:43-47.

26. PEREIRA RN. Relação entre força muscular respiratória e desempenho físico de jogadores de basquetebol em cadeira de rodas. Dissertação (Mestrado) - Universidade metodista de Piracicaba, São Paulo, 2013.

27. PEREIRA RN, et al. Respiratory muscle strength and aerobic performance of wheelchair basketball players. Motriz: Revista de Educação Física, 2016; 22(3): 124-132.

28. ROUANET C, et al. Traumatic spinal cord injury: current concepts and treatment update. Arquivos de neuropsiquiatria, 2017; 75(6): 387-393.

29. SANTOS MVL, et al. Perfil antropométrico e consumo alimentar de atletas de basquetebol em cadeira de rodas da região metropolitana de São Paulo. Revista Brasileira de Nutrição Esportiva, 2016; 10(58): 467-473.

30. SANTOS RMGD, et al. Manovacuometria realizada por meio de traqueias de diferentes comprimentos. Fisioterapia e Pesquisa, 2017; 24(1): 9-14.

31. SERON BB, et al. Analysis of physiological and kinematic demands of wheelchair basketball games-a review. The Journal of Strength \& Conditioning Research, 2019; 33(5): 1453-1462.

32. SKUCAS K, et al. Influence of sport and injury level on mental characteristics of patients with spinal cord injuries. Zhurnal nevrologii i psikhiatrii imeni SS Korsakova, 2014; 114(9): 70-72.

33. VANCINI RL, et al. Avaliação d parâmetros isocinéticos da articulação do ombro de atletas de basquete em cadeiras de rodas. Dissertação de Mestrado. Universidade Federal do Espírito Santo, 2018.

34. WANG X, et al. Effects of Respiratory Muscle Training on Pulmonary Function in Individuals with Spinal Cord Injury: An Updated Meta-analysis. BioMed research international, 2020; 2020: 7530498. 